\title{
Commentary
}

\section{Can Scarring Be Turned Off?}

\author{
Jeffrey M. Davidson \\ From the Department of Pathology, Vanderbilt University and the \\ Medical Research Service, Veterans Affairs Tennessee Valley \\ Healthcare System, Nashville, Tennessee
}

The waxing and waning of sequential events characterize the process of adult tissue repair: hemostasis, inflammation, fibrogenesis, angiogenesis, epithelization, and (in most tissues) scarring. ${ }^{1}$ Much of the analysis of regulation has focused on the activation of these events through local extrinsic signals impinging on transmembrane receptors. Indeed, these signals, whether they are cytokines, growth factors, arachidonic acid metabolites, or even simpler molecules such as nitric oxide, have been observed to undergo dynamic changes that are closely correlated with the activation of repair events, but questions arise. Are these repair processes attenuated merely by the cessation of signaling? Are there feedback mechanisms that govern healing outcomes through alternative pathways? A series of recent observations by Wells et al have begun to strengthen the notion that there are indeed "off" signals that operate during repair. Their current report $^{2}$ on the effects of global CXCR3 deletion suggests that lack of this chemokine receptor results in protracted and incomplete healing followed by a remarkable reemergence of inflammation and scarring several months after the primary skin injury.

\section{What Turns Healing Off?}

Two decades of wound healing investigation have shown that the dynamic changes in wound organization are paralleled by changes in the abundance and/or expression of cytokines and growth factors. The discovery of these signal pathways, beginning with epidermal growth factor (EGF), has lead to the development of diverse strategies to augment such signals where healing is impaired or to attenuate them where healing is excessive. ${ }^{3}$ The initial burst of signaling in acute wounds derives, in large part, from preformed stores in platelets or the extracellular matrix. Beyond the acute phase, recruited inflammatory cells and resident cell populations express an array of mediators that orchestrate the healing process.

Very little has been revealed about the putative inhibitory feedback mechanisms that could either attenuate the pos- itive signals or their response mechanisms to bring about the normal progressive resolution and involution of aggressive granulation tissue into a scar and hence to a healed wound. Several chemical and physical factors have been suggested as part of a negative feedback loop: oxygen (via hydroxylation of Hif- $1 \alpha$ ), mechanical stress (acting through integrins and adhesion complexes), endostatin (and several other matrix protein fragments), angiostatin, angiopoietin 2, and extracellular proteinases.

In a series of publications, the laboratory of Alan Wells has reported its investigations of the wound healing actions of two ligands for the CXCR3 receptor: IP-9 (interferon- $\gamma$ inducible protein-10; CXCL11) and IP-10 (CXCL10). CXCR3 is a $G$ protein-coupled receptor that also recognizes CXCL9 and CXCL4/PF4. This receptor plays a key role in $T$ cell activation and recruitment as well as in allograft destruction. Initial reports from the Wells laboratory showed that endothelial IP-10 inhibited EGF-stimulated fibroblast motility in vitro, ${ }^{4}$ whereas IP-9, produced predominantly in injured epidermis, had the opposite effect on migration of undifferentiated keratinocytes. ${ }^{5}$ These observations led the investigators to propose that the contrasting effects of the CXCR3 ligands in the two tissue compartments could represent a cis-and trans-acting communication system to promote epithelization while limiting the growth factordriven expansion of mesenchymal tissue.

This differential effect of the CXCR3 ligands was attributed to the inhibition of calpain proteinases in fibroblasts, thus preventing fibroblast detachment, and the activation of calpains in keratinocytes, thus promoting keratinocyte deadhesion, presumably when IP-9 binding to CXCR3 drives $\mathrm{Ca}^{2+}$-dependent activation of $\mu$-calpain (ie, calpain 1) via PLC- $\beta 3$ signaling. This activation process would lead to cleavage of several components of the focal adhesion complex at the leading edge of the cell. Furthermore, EGFinduced motility is dependent on ERK-mediated activation of M-calpain (ie, calpain 2). In fibroblasts, however, IP-9 blocked EGF activation of M-calpain and conversely prevented cell detachment at the trailing edge. ${ }^{6}$

Supported by grants from the Department of Veterans Affairs and U.S National Institutes of Health grants AG06528, DK065656, and AR056138. Accepted for publication January 27, 2010

Address reprint requests to Jeffrey M. Davidson, Ph.D., Department of Pathology, C-3321 MCN, Vanderbilt University School of Medicine, Nashville, TN 37232-2562. E-mail: jeff.davidson@vanderbilt.edu. 
These findings led the authors to propose that: (1) juxtacrine actions of epidermal IP-9 could promote epithelial resurfacing while paracrine action inhibited fibroblast and endothelial cell mobilization, and (2) concurrent endothelial cell expression of IP-10 could reinforce the inhibitory process in the dermal/mesenchymal compartment. Subsequently, knockdown of IP-9 expression in the epidermis of transgenic mouse via a keratin 5-driven antisense vector helped to parse out the relative effects of these two CXCR3 ligands. ${ }^{7}$ Epidermal healing was delayed in these mice, and the adjacent superficial dermis showed persistent hypercellularity and reduced organization as long as 60 days after injury. Despite the knockdown of the epidermal IP-9 expression, inflammation was not reduced. The interpretation of these ELR ${ }^{-}$ CXC cytokine (IP-9 and IP-10) knockout experiments could be confounded by the expression of these various ligands in different tissue compartments as well as the acute release of an additional CXCR3 ligand, PF4/CXCL4, after tissue injury.

In the global CXCR3 knockout mouse, wound healing is markedly impaired and delayed, with notable defects in the organization of the epidermis and its basement membrane up to 90 days after injury. ${ }^{8}$ The report in this issue $^{2}$ describes the seeming reversal of the healing process when observation of wounds in these null mice continued out to 180 days. The authors observed epidermal thickening, poor organization of the dermal extracellular matrix, and concomitant defects in mechanical properties, which represent properties similar to human hypertrophic scars. The revival of an inflammatory response in the absence of infection shows that multiple aspects of the immune system were also affected by the loss of CXCR3. These findings suggest that the CXCR3 pathway, at least in mice, may be critical to involution and resolution of cutaneous healing.

There are certainly parallels and contrasts between the nature of the healing response in this model and the classical examples of excess cutaneous scarring in the human-hypertrophic scars and keloids. However, the persistence of incompletely reorganized wound sites and the regression of wound organization in this knockout model are quite remarkable.

It has been extremely difficult to find and use animal models to understand the cutaneous scarring process, although a wide variety of fibroses can be generated in cardiac, renal, pulmonary, or hepatic tissue. The only alternatives to date have been large excisions in the rabbit ear, the red Duroc pig, repeated bleomycin infiltration into mouse skin, and, most recently, progressive mechanical distension of incisional wounds in the mouse. It is possible that hypertrophy in these wounds is driven by a combination of an inappropriate mechanical environment and inflammatory mediators.

\section{CXCR3, IFN- $\gamma$, and Fibrosis}

The ELR ${ }^{-}$CXC chemokines are induced by interferon (IFN)- $\gamma$, and they play roles in many aspects of inflammation and repair. The CXCR3 receptor is expressed on
T-cells, NK cells, monocytes, dendritic cells, endothelial cells, fibroblasts, and keratinocytes. CXCR3 ligands act as potent leukocyte chemoattractants, and they are likely to modulate the abundance of NK and other T-cell populations at sites of acute and chronic injury. These chemokines are also known to inhibit angiogenesis through interaction with endothelial CXCR3. ${ }^{9}$ In the mouse model of bleomycin-induced pulmonary fibrosis, IP-10 administration reduced the severity of fibrosis, ${ }^{9}$ and knockout of CXCR3 led to a more severe fibrotic response and increased mortality without increased inflammatory cell recruitment. ${ }^{10}$ This report also proposed that early, selfamplifying induction of IFN- $\gamma$ expression by $\mathrm{CXCR} 3$ signaling (via PF4/CXCL4) could be a critical step in limiting the fibrotic response. Recent analysis of an IP-10 knockout in a cardiac infarct model showed a delay in scar contraction, rapid thinning of the ventricular wall, and a shift in the composition of the infiltrating cell populations. ${ }^{11}$ These and many other studies indicate that signaling through the CXCR3 can influence many other cell types in the immune and inflammatory system. Thus, it is likely that global knockout of CXCR3 affects the tissue repair process at many levels and stages of repair. The delayed reappearance of a chronic wound phenotype in the present report may well reflect a contribution of the CXCR3 signal pathway in the immune system rather than resident epithelial and mesenchymal cells.

\section{Alternative Pathways}

The global nature of the CXCR3 knockout could certainly have an impact on cell types other than resident wound cells. The current work discusses potential effects on recruitment of endothelial and mesenchymal progenitors from the circulation, but the known participation of the CXCR3 circuitry in lymphocyte/T-cell and perhaps macrophage recruitment should not be overlooked. ${ }^{12}$ Both cell types are involved in the wound repair process. In the fibrotic lung, the immunomodulatory aspects of CXCR3 have been the predominant focus. The striking return of an inflammatory response 6 months after wounding is not typical of the hypertrophic scar. This phenomenon could well involve aberrant regulation of the immune system rather than further alteration of resident cell behavior.

Absent a cell-specific knockout of CXCR3, the strongest evidence for the importance of epidermal modulation of scar involution and remodeling comes from data obtained with epidermal-driven (keratin 5) overexpression of an IP-9 antisense construct. The current study provides data that partial suppression of IP-9 produces similar morphological effects, even at some distance below the epidermis. This finding does suggest that, at least in the mouse, CXC11/CXCR3 interaction is the dominant mechanism for wound regression.

Hypertrophic scarring is a major complication of severe burn injuries, and burn surgeons have long been aware of the need for epithelial resurfacing to suppress the formation of excess connective tissue..$^{13}$ The predominant modes of action of the CXCR3 system in formed elements appear to be cell motility and (endothelial) ap- 
optosis. It is not certain from the present studies that these processes alone could account for the markedly reduced collagen content of wounds in the CXCR3-null mouse. A number of fibrotic mechanisms besides fibroblast motility have been proposed, including mechanical constraint, regulation of matrix metalloproteinases, and alteration of fibrogenic cytokine profiles. Other investigations of keratinocyte-fibroblast interactions have implicated other soluble mediators, including transforming growth factor (TGF)- $\beta,{ }^{14-16}$ fibroblast growth factor, interleukin (IL)-1, IL-7, ${ }^{9}$ and stratifin ${ }^{17-19}$ as agents that can modify the fibroblast phenotype. Many of these factors affect either the balance between synthesis and degradation of collagen or the rate of cell proliferation. It would be useful to undertake parallel studies, especially in clinical material, of the effects of the ELR ${ }^{-} \mathrm{CXCL}-\mathrm{CXCR} 3$ axis, these alternative signals, and the relative importance of their expression during normal and pathological wound healing.

\section{CXCR3 and Fibroplasia}

Previous studies in the CXCR3-null mouse certainly agree with the role of this receptor in limiting fibroplasia. It has been exceedingly difficult to induce persistent hypertrophic scarring in animal skin by any means. The long-term response of the CXCR3-null wound does reflect several features of the hypertrophic scar, although it does not appear raised above the plane of the skin or hypervascularized. The CXCR3-null animals showed significantly increased, progressive, interstitial fibrosis without persistent inflammation in the pulmonary bleomycin model, whereas acute inflammation created patchy fibrosis in wild-type mice. ${ }^{10}$ These authors proposed that the suppression of an early burst of IFN- $\gamma$ activity could have interrupted a positive feedback loop in which CXCL10/ IP-10 is up-regulated. Indeed, administration of IFN- $\gamma$ or adoptive transfer of $\mathrm{CXCR}^{+}$leukocytes was able to suppress the fibrotic response, and immunoneutralization of IFN- $\gamma$ was fibrogenic. Although this study certainly supports the central role of $\mathrm{CXCR} 3$, it suggests that pathways other than calpain activation may contribute to suppression of the fibrotic response. Epithelization and the contribution of epithelial IP-9 were not considered in this study; however, there is a clear relationship between lung epithelial (basal lamina) integrity and the progression of interstitial fibrosis. ${ }^{9,20}$

A similar study of renal fibrosis via unilateral urethral obstruction showed that both CXCR3 and IP-10 were up-regulated during the fibrotic response. ${ }^{21} \mathrm{As}$ in the skin and lung, both CXC3R gene knockout and IP-10 immunoneutralization promoted interstitial fibrosis, with decreased hepatocyte growth factor and increased TGF- $\beta 1$ expression. Neither macrophage nor T-cell infiltration seemed to be affected by IP-10 neutralization. In contrast to the epidermal IP-9 signaling, an immortalized tubular epithelial cell line expressed IP-10 and CXCR3 in response to IFN- $\gamma$ stimulation, and this response was amplified in the presence of TNF- $\alpha$. These authors also interpreted the protective effects of IP-10/CXCR3 as not involving inflammatory cell recruitment. The shift from epithelial IP-9 to IP-10 in the kidney may reflect the tendency of proximal tubular epithelial cells to undergo epithelial mesenchymal transformation, particularly under the influence of TGF- $\beta$.

The current article places significant emphasis on the interaction of CXCR3 with the intracellular focal adhesion apparatus via calpains 1 and 2. Cell adhesion and traction are also likely to be involved in a purely mechanical model of hypertrophic scarring. ${ }^{22}$ However, these investigators focused on an anti-apoptotic aspect, suggesting that sustained mechanical stress promoted increased cell density by inhibition of an Akt-dependent mechanism. The authors further suggest that fibroblast number is the predominant fibrotic mechanism. The canonical CTGF-TGF $\beta$-SMAD pathway has certainly been invoked in related systems. However, if CXCR3-null fibroblasts have a significant defect in calpain activation, the resultant increased stability of focal adhesions might increase mechanotransduction and thereby matrix synthesis. In addition, other signals, including mechanical stress, can activate calpain and thereby modulate signaling mediated by the focal adhesion complex.

The observations presented in the accompanying article certainly reinforce the concept that wound healing gets both turned on and turned off. CXCR3 agonists and antagonists, if locally delivered, could therefore become an important therapeutic tool for diminishing the exuberant accumulation of connective tissue or overcoming a premature suppression of repair. It will be quite important to understand how the present observations tie into human pathologies of tissue repair and fibrosis.

\section{References}

1. Gurtner GC, Werner S, Barrandon Y, Longaker MT: Wound repair and regeneration. Nature 2008, 453:314-321

2. Yates CC, Krishna P, Whaley D, Bodnar R, Turner T, Wells A: Lack of CXC chemokine receptor 3 (CXCR3) signaling leads to hypertrophic and hypercellular scarring, Am J Pathol 2010, 176:1743-1755

3. Werner S, Grose R: Regulation of wound healing by growth factors and cytokines. Physiol Rev 2003, 83:835-870

4. Shiraha H, Glading A, Gupta K, Wells A: IP-10 inhibits epidermal growth factor-induced motility by decreasing epidermal growth factor receptor-mediated calpain activity. J Cell Biol 1999, 146:243-254

5. Satish L, Yager D, Wells A: Glu-Leu-Arg-negative CXC chemokine interferon gamma inducible protein-9 as a mediator of epidermaldermal communication during wound repair. J Invest Dermatol 2003, 120:1110-1117

6. Allen FD, Asnes CF, Chang P, Elson EL, Lauffenburger DA, Wells A Epidermal growth factor induces acute matrix contraction and subsequent calpain-modulated relaxation. Wound Repair Regen 2002, 10:67-76

7. Yates CC, Whaley D, A YC, Kulesekaran P, Hebda PA, Wells A: ELR-negative CXC chemokine CXCL11 (IP-9/I-TAC) facilitates dermal and epidermal maturation during wound repair. Am J Pathol 2008, 173:643-652

8. Yates CC, Whaley D, Hooda S, Hebda PA, Bodnar RJ, Wells A Delayed reepithelialization and basement membrane regeneration after wounding in mice lacking CXCR3. Wound Repair Regen 2009, $17: 34-41$

9. Strieter RM, Gomperts BN, Keane MP: The role of CXC chemokines in pulmonary fibrosis. J Clin Invest 2007, 117:549-556

10. Jiang D, Liang J, Hodge J, Lu B, Zhu Z, Yu S, Fan J, Gao Y, Yin Z, 
Homer R, Gerard C, Noble PW: Regulation of pulmonary fibrosis by chemokine receptor CXCR3. J Clin Invest 2004, 114:291-299

11. Bujak M, Dobaczewski M, Gonzalez-Quesada C, Xia Y, Leucker T, Zymek P, Veeranna V, Tager AM, Luster AD, Frangogiannis NG Induction of the CXC chemokine interferon-gamma-inducible protein 10 regulates the reparative response following myocardial infarction. Circ Res 2009, 105:973-983

12. Romagnani $\mathrm{P}$, Lasagni L, Annunziato F, Serio M, Romagnani S: CXC chemokines: the regulatory link between inflammation and angiogenesis. Trends Immunol 2004, 25:201-209

13. Deitch EA, Wheelahan TM, Rose MP, Clothier J, Cotter J: Hypertrophic burn scars: analysis of variables. J Trauma 1983, 23:895-898

14. Mukhopadhyay A, Tan EK, Khoo YT, Chan SY, Lim IJ, Phan TT: Conditioned medium from keloid keratinocyte/keloid fibroblast coculture induces contraction of fibroblast-populated collagen lattices. Br J Dermatol 2005, 152:639-645

15. Phan TT, Lim IJ, Aalami O, Lorget F, Khoo A, Tan EK, Mukhopadhyay A, Longaker MT: Smad3 signalling plays an important role in keloid pathogenesis via epithelial-mesenchymal interactions. J Pathol 2005, 207:232-242

16. Xia W, Phan TT, Lim IJ, Longaker MT, Yang GP: Complex epithelialmesenchymal interactions modulate transforming growth factor-beta expression in keloid-derived cells. Wound Repair Regen 2004, 12:546-556
17. Medina A, Ghaffari A, Kilani RT, Ghahary A: The role of stratifin in fibroblast-keratinocyte interaction. Mol Cell Biochem 2007, 305: 255-264

18. Lam E, Kilani RT, Li Y, Tredget EE, Ghahary A: Stratifin-induced matrix metalloproteinase- 1 in fibroblast is mediated by c-fos and p38 mitogen-activated protein kinase activation. J Invest Dermatol 2005, 125:230-238

19. Ghahary A, Karimi-Busheri F, Marcoux Y, Li Y, Tredget EE, Taghi Kilani R, Li L, Zheng J, Karami A, Keller BO, Weinfeld M: Keratinocytereleasable stratifin functions as a potent collagenase-stimulating factor in fibroblasts. J Invest Dermatol 2004, 122:1188-1197

20. Vracko R: Basal lamina scaffold-anatomy and significance for maintenance of orderly tissue structure. Am J Pathol 1974, 77:314-346

21. Nakaya I, Wada T, Furuichi K, Sakai N, Kitagawa K, Yokoyama H, Ishida $Y$, Kondo T, Sugaya T, Kawachi H, Shimizu F, Narumi S, Haino M, Gerard C, Matsushima K, Kaneko S: Blockade of IP-10/CXCR3 promotes progressive renal fibrosis. Nephron Exp Nephrol 2007, 107:e12-21

22. Aarabi S, Bhatt KA, Shi Y, Paterno J, Chang El, Loh SA, Holmes JW Longaker MT, Yee H, Gurtner GC: Mechanical load initiates hypertrophic scar formation through decreased cellular apoptosis. FASEB J 2007, 21:3250-3261 